Editorial

\title{
Special Issue on Image and Video Quality Assessment
}

Quality assessment and control of images and videos from the input (capture device) to the final output (display and its environment) presented to the human viewer is essential for image/video applications and services.

PSNR and other related measures have long been the most popular quality criteria used by engineers and researchers to evaluate and optimize the performances of digital image and video processing schemes. However, these simpleto-compute measurements are not in good agreement with human visual quality judgment. Therefore, there is an established need for efficient Image and Video Quality Assessment (IQA/VQA) schemes.

Over the past two decades, objective image and video quality assessment methods have been extensively studied, and many criteria have been proposed. Depending on the application and the type of information accessible, IQA/VQA can be classified into three types: Full Reference (FR) metrics, Reduced Reference (RR) metrics, and No Reference (NR) metrics. All these types of visual quality metrics are now considered for standardization by various groups, including the Video Quality Experts Group (VQEG) for video and JPEG Advanced Image Coding (AIC) for images.

Even if improvements have been achieved compared to PSNR, there is a need for more efficient and generic IQA/VQA algorithms that are more robust to the image $\&$ video content and also to the different types of impairments introduced by the degrading systems.

The aim of this special issue is to provide an overview of state-of-the-art IQA/VQA methods and to address new developments towards various directions. The issue starts with an invited paper that surveys an area of great current research interest, namely NR QA. It is followed by another survey paper, which examines audiovisual
QA. The next two papers each propose a novel image quality metric, one based on gradient profiles, and the other on structural similarity. They are followed by a paper on perceptual deblocking. Video quality is the topic of the last two papers, one investigating spatio-temporal interactions in VQA, and the other the impact of visual attention on VQA.

The invited paper by S. S. Hemami and A. R. Reibman is a survey on the topic of "No-reference image and video quality estimation: Applications and human-motivated design." Their paper proposes a three-stage framework for noreference quality estimators that encompasses the range of potential use scenarios and allows knowledge of the human visual system to be incorporated throughout. It also surveys the measurement stage of the framework, considering methods that rely on bitstream, pixels, or both. By exploring the accuracy requirements of potential uses as well as evaluation criteria to stress-test a quality metric, the paper sets the stage for the IQA/VQA community to make substantial future improvements to the challenging problem of NR quality estimation.

The paper "Perceptual-based quality assessment for audio-visual services: A survey" by J. You, U. Reiter, M. M. Hannuksela, M. Gabbouj, and A. Perkis addresses the important issue of joint audio-visual quality assessment. While many studies have targeted audio and video quality assessment separately, fundamental research is required on multi-modal perception to better understand the mutual influence between auditory and visual stimuli. This paper reviews subjective quality assessment methodologies for audio-visual signals, and surveys perceptual-based audio-visual quality metrics.

The paper "No-reference perceptual image quality metric using gradient profiles for JPEG2000" by $\mathrm{L}$. Liang, S. Wang, J. Chen, S. Ma, D. Zhao, and W. 
Gao deals with one of the major difficulties in NR $Q A$, which is that certain features of natural images can be ambiguous with artifacts. They tackle this problem using statistical information on image gradient profiles and propose a novel quality metric for JPEG2000 images. The key part of the metric is a histogram representing the sharpness distribution of the gradient profiles, from which a blur metric is derived that is insensitive to inherently blurred structures in the natural image. Furthermore, a ringing metric is defined based on ringing visibilities of regions associated with the gradient profiles. The combined model is robust to various types of image content and achieves a performance competitive with state-of-the-art metrics.

The contribution by $\mathrm{C}$. $\mathrm{Li}$ and A. C. Bovik on "Content-partitioned structural similarity index for image quality assessment" deals with an attempt to take into account image content within the framework of an SSIM-based FR image quality metric. Based on the fact that different image regions have different perceptual significance relative to quality, the authors incorporate a fourcomponent image model that classifies each of the image's local regions into one of four types according to edge and smoothness properties: changed edge region, preserved edge region, textured region, smoothed region. Local SSIM scores are then weighted by region type.

The paper "Deblocking filtering method using a perceptual map" by A. Chetouani, G. Mostafaoui, and $\mathrm{A}$. Beghdadi proposes an image deblocking method, which aims to reduce blocking artifacts by analyzing their visibility. A perceptual map is used as input to a recursive filter in order to reduce the blocking effect. The obtained results compare favorably with a recent method.

The paper by Q. Huynh-Thu and M. Ghanbari, "Modelling of Spatio-Temporal Interaction for Video Quality Assessment", looks at the way spatial and temporal distortions interact and how this affects overall perceived video quality. More specifically, the paper shows that an artifact in one modality impacts the perceived quality of the other modality. It is found that interaction is more significant at high quality, and that spatial quality contributes more to the overall quality. An objective model is introduced to predict overall video quality by integrating spatial and temporal qualities. This model is then validated with subjective data.
The paper by O. Le Meur, A. Ninassi, P. Le Callet and D. Barba on "Overt visual attention for freeviewing and quality assessment tasks - Impact of the regions of interest on a video quality metric" concludes our special issue. It describes two eye tracking experiments that were designed to investigate how people watch video during freeviewing and quality assessment tasks. The comparison between gaze allocations indicates that the latter has a moderate impact on the visual attention. The level of distortion does not significantly alter the oculomotor behavior. Finally, an objective full-reference video quality metric is adapted to include saliency-based distortion pooling, which is shown to result in no significant improvements of prediction performance.

In conclusion, this special issue presents a peek at recent advancements in the fields of image and video quality assessment. We trust that the reader will enjoy and benefit from the collection of papers herein.

We would like to thank all the authors for their excellent contributions. We are also grateful to all reviewers, whose comments and suggestions helped improve the quality of the papers. Finally, we extend our thanks to Editor-in-Chief Murat Tekalp for his help in putting this special issue together.
Stefan Winkler
Cheetah Technologies, San Jose, USA
swinkler@cheetahtech.com

Frederic Dufaux

Ecole Polytechnique Fédérale de Lausanne,

Switzerland

frederic.dufaux@epfl.ch

Dominique Barba

Université de Nantes, France dominique.barba@univ-nantes.fr

Vittorio Baroncini

Fondazione Ugo Bordoni, Rome, Italy vittorio@fub.it 


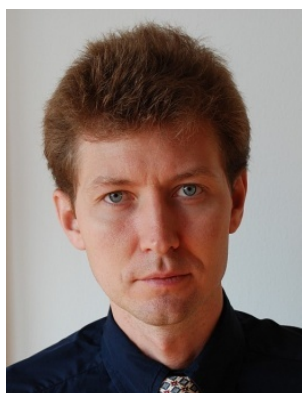

Stefan Winkler holds a M.Sc. degree in Electrical Engineering from the University of Technology in Vienna, Austria, and a Ph.D. degree from the Ecole Polytechnique Fédérale de Lausanne

(EPFL),

Switzerland. He is currently Chief Scientist at Cheetah Technologies. Prior to that, he was Principal Technologist at Symmetricom and Chief Scientist at Genista, which he co-founded in 2001. He has also held assistant professor positions at the National University of Singapore (NUS) and the University of Lausanne, Switzerland.

Dr. Winkler has 15 years of experience in the areas of media processing and quality assessment. He has published more than 60 papers and is the author of the book "Digital Video Quality". He serves as an Associate Editor for IEEE Transactions on Image Processing.

He has been an active contributor to the Video Quality Experts Group (VQEG) since it was founded in 1997 and is currently co-chair of the QoE Metrics Activity Group of the Video Services Forum (VSF). He has also contributed to the ATIS IPTV Interoperability Forum (IIF). His research interests include image and video quality assessment, perception, computer vision, and human-computer interaction.

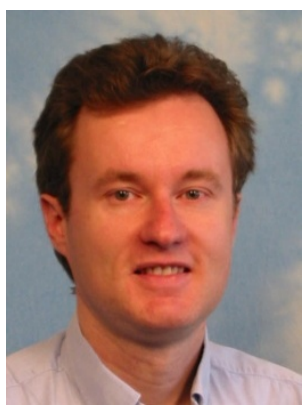

Frédéric Dufaux received his M.Sc. in physics and Ph.D. in electrical engineering from the Swiss Federal Institute of Technology (EPFL), Lausanne, Switzerland, in 1990 and 1994 respectively.

Frédéric has over 20 years of experience in research. In 1992, he was a visiting researcher at AT\&T Bell Laboratories. In 1994 and 1995, he was a Postdoctoral Fellow at the Massachusetts Institute of Technology. From 1995 till 2001, he was a senior member of research staff at Digital Equipment Corp. / Compaq Computer Corp. In 2001, he joined Genimedia SA as a principal solutions architect. Since 2003, he is a scientific senior research staff at EPFL. From 2003 to 2009, he was also chief scientist of Emitall Surveillance SA.
Frédéric has been involved in the standardization of digital video and imaging technologies for more than 15 years, actively participating in MPEG and JPEG committees. He is currently co-chairman of JPEG2000 over wireless (JPWL) and JPSearch. He is the recipient of two ISO awards.

His research interests include image and video coding, distributed video coding, compressed sensing, subjective visual quality assessment, objective quality metrics, video surveillance, privacy protection, multimedia security, video transmission over wireless network, and multimedia content search and retrieval.

Frédéric is the author or co-author of more than 90 research publications and holds 10 patents in the field of media technologies.

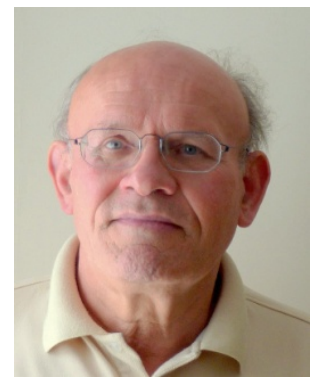

Dominique Barba is Professor Emérite at the University of Nantes since 2006. He received the Master degree in Physics Sciences from the University of Reims, France, in 1968, the Ph.D degree in Telecommunications from the University of Rennes 1, France, in 1973, and the Doctorat es Sciences Mathématiques (Computer Sciences) from the University of Paris VI, France, in 1981, respectively.

From 1968 to 1973, he was a Lecturer at the University of Rennes 1, working in the field of Ultrasonic Digital Signal Processing. From 1973 to 1984, he was an Associated Professor at the Institut National des Sciences Appliquées (INSA) in Rennes, working in the field of Digital Image Processing with psycho-visual quality criterion, with application to image restoration and to image \& video compression. He worked also in the field of image synthesis with real time constraints.

He was attached in 1984-1985 to the French national research Center CNET/CCETT in charge of image and video compression activities.

He joined as a full Professor a new Graduate School of Engineering (IRESTE) created at the University of Nantes in 1986, and transformed into Ecole Polytechnique de l'Université de Nantes in 2000. He launched new research activities in Nantes in the field of digital image \& video processing and was personally involved in research on Pattern Recognition and Image 
Analysis, Machine Vision, Image and Video representation and coding with special interest to high level image \& video data description and compression for multimedia communication, human visual modeling for image perception and recognition. During this period, he held a number of responsibility positions: head of a research group, deputy director of IRCCYN (a large research laboratory attached to the CNRS, a French research agency), head of the research activities of IRESTE. He served also as scientific expert in many Governmental Research Networks.

$\mathrm{He}$ is author or co-author of hundreds of papers in scientific journals or international conferences and holds 14 patents in the field of image technologies and computer vision.

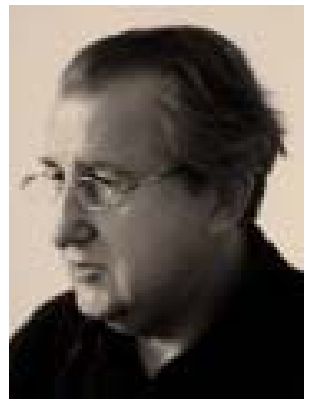

Vittorio Baroncini is Senior Researcher at the Fondazione Ugo Bordoni (FUB) in Rome, Italy. He began work in 1976 at the ITT R\&D Laboratory of Pomezia (Italy). In 1984, he joined the "Instituto Superiore delle Poste e Telecomunicazioni," the technical branch of the Italian Government's Telecommunication Ministry. In 1986, he joined FUB's TV group.

At the same time, he began participating in international standards work, by contributing to the work of the CCIR (now ITU-R) Committee. He was active in the SC29-WG11 (MPEG) group of the International Standards Organization (ISO), dealing with MPEG-2 and chairing all he MPEG-4 AVC/SVC competition and verification tests. Thanks to the participation to the European projects QUOVADIS and MOSQUITO, he became an expert in objective quality video assessment.

$\mathrm{He}$ is the designer of a number of new subjective evaluation methods such as Single Stimulus with Multiple Repetition, Double Stimulus Hidden Reference, Service Acceptance, and the test method to evaluate the vertical resolution of $35 \mathrm{~mm}$ film. He designed and conducted the first quality evaluation test of D-Cinema.

Recently he coordinated and designed the biggest formal subjective testing effort ever done. The HeVC (High efficiency Video Coding) Call for proposal (coming from an ISO/ITU joint effort) received 27 submissions from all over the world.
Following to this call the proponents produced 4205 video clips that were subjectively tested at three international laboratories (EPFL, EBU and FUB) running a total of 134 test sessions, with more than 850 viewing subjects that produced around than 300.000 scores.

Currently he is on the steering board of ITU-R Study Group 6 (Broadcasting Services), as vicechair of WP6C (Programme production and quality assessment) and an active member of VQEG. He is the technical responsible for the HDTV and DCinema projects in FUB. He is author or co-author of many papers to conferences and journals as well as a book on MPEG-4. 